\title{
Guessing strategy constraints in the Bransford-Franks paradigm
}

\author{
LANCE M. POLLACK and STUART KATZ \\ University of Georgia, Athens, Georgia 30602
}

\begin{abstract}
The Bransford-Franks (1971) integration hypothesis and an alternative guessing strategy hypothesis were tested one against the other by varying the constraint under which the "atoms" comprising stimulus items could be arranged. Results showed that as constraint decreased, the slopes of the best-fitting linear curves for item "complexity" (i.e., number of atoms in an item) reversed direction, a finding which could only have been predicted by the guessing strategy hypothesis.
\end{abstract}

Bransford and Franks (1971) proposed that when individuals are exposed to complex stimulus items, they select related components of the items and integrate them into wholistic concepts. What is subsequently remembered, according to their model, is the integrated concept itself and not the specific items. In the standard Bransford-Franks paradigm, integration is demonstrated, presumably, by the observed linear relation between "recognition confidence" (a measure of recognition) and item "complexity" (the number of components contained in any recognition item). The greater the complexity of a recognition item, the closer that item approximates the integrated concept and, thus, the higher its recognition. However, studies replicating the Bransford-Franks experiments but varying instructions (Katz, 1973; but see also Flagg, Potts, \& Reynolds, 1975), complexity of stimulus items (Holland \& Katz, Note 1), and method of constructing such items (Reitman \& Bower, 1973) showed the linear relation could be made to disappear, though the integration hypothesis would not always have predicted this. Therefore, an alternative explanation was offered in the form of a simple guessing strategy hypothesis (Katz \& Gruenewald, 1974; Katz, Atkeson, \& Lee, 1974). According to this explanation, given a recognition item containing a certain number of elementary components or "atoms" (the number of atoms contained in an item will hereafter be referred to as "combination size"), the greater the number of possible combinations of atoms for that combination size, the lower will subjects estimate the probability of their having seen that particular item in acquisition. The lower this estimate, in turn, the lower the recognition.

Thus, the linear effect may be understood as an artifact of a particular rule used to combine atoms into items of varying combination size, because such a rule guarantees relatively small numbers of arrangements of atoms for relatively large combination sizes. The

This paper is sponsored by Robert B. Payne, who takes full editorial responsibility for its content. rule, in other words, provides a greater constraint on these numbers. Rules with less constraint, however, are also possible. Such rules can be used to maintain the same number of one-atom items (ONEs) while drastically increasing the number of possible combinations for two-atom items (TWOs), three-atom items (THREEs), four-atom items (FOURs), etc. The purpose of the present study is to vary systematically the constraint in the combination rules to determine its effect on the linear relationship. This information can then be used to determine which hypothesis, integration or guessing strategy, best fits the data. The reasoning is as follows.

According to the integration hypothesis, when constraint is reduced the atoms comprising the total concept interrelate in many different ways, and this tends to block the integration of the atoms into unified concepts. The lack of integration should, therefore, produce comparable ratings for all recognition items, regardless of combination size, because each of these items, instead of becoming part of a larger whole, stands separate and unique (Bransford \& Franks, 1973). Thus, as constraint in the combination rules decreases, the integration hypothesis predicts a change from a linear relationship between recognition and combination size to a "zeroslope," or flat, relationship in which recognition ratings are comparable for all combination sizes.

According to the guessing strategy hypothesis, on the other hand, less constraint means an increase in the number of possible combinations for items of larger combination size. This increase lowers the probability that an item of larger combination size could be selected from all items of that size and will, if the hypothesis is true, lower recognition for that item. In fact, as constraint decreases, the linear effect should be reversed, so that in a condition involving minimal constraint, ONEs should show the highest ratings, while successively larger combination sizes should show successively lower recognition scores (e.g., Reitman \& Bower, 1973).

In sum, decreasing constraint in the rules by which atoms are combined should, if the integration hypothesis is true, show recognition curves that tend toward flatness across combination size, while the guessing strategy 
hypothesis should show a gradual reversal in the slopes of these curves.

\section{METHOD}

\section{Subjects}

Subjects were 72 undergraduate students enrolled in introductory psychology courses at the University of Georgia.

\section{Materials}

All stimulus materials were constructed from a universe of 12 atoms: $1234 \mathrm{ABCDWXYZ}$.

In the most constrained condition (Rule 1), a virtual replication of the original procedure for constructing the stimuli, the universe of atoms was divided into three concepts of four atoms each: 1234, ABCD, WXYZ. According to Rule 1, (1) all items must maintain the left-to-right order established in the universe, (2) no item can obtain atoms from more than one of the concepts, and (3) no atom can be repeated within the same item. Thus, for example, the concept 1234 had the following possible arrangements: $1,2,3,4,12,13,14,23,24,34,123$, $124,134,234,1234$. The acquisition list was composed of all possible arrangements for all three concepts. The items generated by this rule were presented in random order, but with the limitation that one item could not be followed by another from the same concept. The recognition list was composed of the same 45 items used in acquisition. The list was presented in a different random order, again with the same limitation that no item be followed by another from the same concept.

A second condition (Rule 2) eliminated boundaries between concepts, thus creating a single concept of 12 atoms. The order of atoms was maintained, as was the prohibition of repetition. Thus, an item like, for example, $\mathrm{AB} 12$ is permissible under this rule, whereas it is not under Rule 1 . The acquisition list was composed of 12 ONEs, 12 TWOs, 12 THREEs, and 12 FOURs presented in random order. The recognition list contained the same 48 items presented in acquisition, but in a different random order.

A third condition (Rule 3 ) eliminated boundary and order but, again, repetition was prohibited. For example, an item like B1A2 was permissible here, whereas it was not under Rules 1 and 2. The acquisition list was composed of 12 each of ONEs, TWOs, THREEs, and FOURs presented in random order. The recognition list was composed of the same items, but in a different random order.

A fourth condition (Rule 4) eliminated boundary and order and allowed repetition as well; thus, it was the least constrained of the four rules. For example, an item like BA22 was permissible here. Once again the acquisition list was composed of 12 each of ONEs, TWOs, THREEs, and FOURs presented in a random order. The recognition list was composed of the same 48 items in acquisition, but presented in a different random order.

The items in each list were typed on a deck of $4 \times 6$ in. $(10.2 \times 15.3 \mathrm{~cm})$ white index cards, one item per card. The items were composed of printed black letters and numbers $.4 \mathrm{~cm}$ tall.

\section{Procedure}

Acquisition involved an incidental learning task. Subjects, in groups of two or three, were shown a card containing one item for $3 \mathrm{sec}$. Each item was followed by a 5 -sec interval in which subjects counted aloud the number of fingers on the experimenter's right hand. After the interpolated task, subjects wrote down the item they had just seen. This procedure was repeated for each item on the acquisition list. Appropriate counterbalancing was employed to control for possible order effects in list presentations. When the list was completed, subjects rested for $5 \mathrm{~min}$. During acquisition, no mention was made of any recognition test.
During recognition, subjects were told that a new list of items would be shown to them and that these items were closely related to the list of items presented in acquisition. Their task was to indicate which items they had actually seen before and which ones they had not. For each item, subjects indicated whether or not they recognized it by first marking a YES-NO scale and then a 5-point confidence scale ranging from "very low" to "very high" confidence. This procedure was repeated for each of the recognition items.

\section{RESULTS}

The dependent measure used in the present experiment was mean "recognition confidence" as measured by a 10-point scale ranging from +5 (very confident that the item was seen before) to -5 (very confident that the item was not seen before). In each condition, ratings were pooled for FOURs, THREEs, TWOs, and ONEs. Figure 1 shows the mean recognition confidence ratings for each combination size across rules.

The data were cast into a two-factor repeatedmeasures design, with rule (Rules $1,2,3,4$ ) and combination size (FOURs, THREEs, TWOs, ONEs) as the factors. The overall analysis of variance yielded significant main effects for rules $[F(3,68)=6.29, p<.001]$ and combination size $[\mathrm{F}(3,204)=11.62, \mathrm{p}<.001]$, as well as a significant interaction between the two factors $[F(9,204)=11.07, p<.001]$. A trend analysis was carried out on the same data and a significant interaction was found between rule and the linear trend for combination size $[F(3,68)=16.33, p<.001]$. As Figure 1

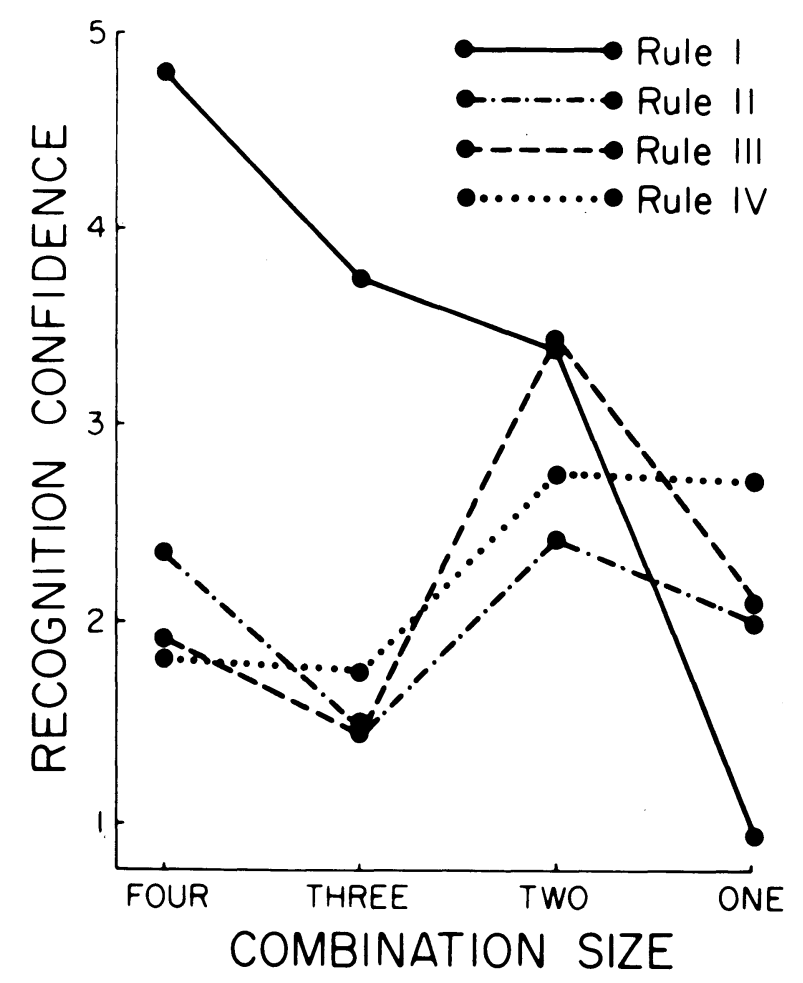

Figure 1. Mean recognition confidence ratings as a function of rule and combination size 
shows, this interaction occurred because of a progressive decline in the recognition confidence scores for large combination sizes and an increase for small combination sizes.

Since a test of the integration vs guessing strategy hypotheses depends on whether, as rule constraint decreases, the slope of the best linear fit of the recognition confidence curve actually reverses direction, additional tests were carried out to determine if this happened. Coefficients of orthogonal polynomials were used to change each subject's scores across combination size into a single linear transform. To determine whether these transforms differed from zero, $t$ tests were then used. The tests showed that the scores for Rule 1 were significantly different from zero in the negative direction $[\mathrm{t}(17)=-7.932, \mathrm{p}<.001]$, thus replicating studies by Bransford and Franks (1971) and Reitman and Bower (1973). The scores for Rule 2 did not differ from zero [ $\mathrm{t}(17)=-.032, \mathrm{p}<.05]$, while the scores for Rules 3 and 4 differed from zero in the positive direction $[\mathrm{t}(17)=2.149, \mathrm{p}<.05$ and $t(17)=1.796, p<.05$, respectively $]$. All tests were one-tailed.

\section{DISCUSSION}

The purpose of this study was to find out which of two possible explanations of the linear effect, integration or guessing strategy, is the correct one. This was done by creating four conditions within the general framework of the BransfordFranks (1971) paradigm, each of which differed in the rule by which the atoms comprising stimulus items could be arranged. In effect, each rule differed in the degree of constraint in the number of possible arrangements as defined by order, boundary, and repetition. Constraint was greatest for Rule 1 (the rule most similar to the rule used in the original research) and thereafter decreased systematically for Rules 2, 3, 4, respectively. According to the integration hypothesis, recognition confidence curves should show a decrease in linearity as we move from Rule 1 to Rule 4, the limit being a flat or zero-slope curve. The guessing strategy hypothesis, on the other hand, predicts that the curves will show a reversal of slopes, from a negative slope for Rule 1 to increasingly positive slopes for Rules 2,3 , and 4 .

The results supported the guessing strategy hypothesis. A trend analysis revealed a significant interaction between type of rule and the linear trend for combination size. As Figure 1 shows, there is a replication of the linear effect for Rule 1 and a progressive reversal of the effect for Rules 2,3 , and 4 . The reversal was confirmed statistically by $t$ tests of the linear transforms of subjects' scores. The tests showed that the slope for Rule 1 was significantly different from zero in the negative direction, and the slopes for Rules 3 and 4 were different from zero in the positive direction.

Though the results support the guessing strategy hypothesis, the pattern of results is not as strong as might be expected if the subjects had based their recognition confidence scores literally on the probabilities of occurrence of items across combination size for each of the rules. For example, in Rule 1 the number of possible arrangements for TWOs was the largest; therefore, the pattern of results should not have been linear, nor even monotonic. Furthermore, Rules 2, 3, and 4 should all have produced reverse linear effects, and the slopes for each condition should have grown steeper as constraint decreased. Since the slope of the Rule 2 condition did not differ from zero, and since additional individual comparisons among the rule conditions did not show a significant difference between Rules 3 and $4(p<.05)$, a literal interpretation of the guessing strategy hypothesis is not supported by the data. Perhaps the best way to treat the formal guessing strategy model, therefore, is as a heuristic. Since subjects are not probability theorists nor high-speed computing devices (both of which would be required for a perfect match between the formal description and the data), they would not be expected to follow the model explicitly. Rather, it may be supposed that the results reflect subjective estimates that only approximately coincide with the formal model. Thus, the next step in research of this type would be to gather data on such estimates and show a correspondence between such data and the results observed here.

\section{REFERENCE NOTE}

1. Holland. M. F., \& Katz. S. The role of a guessing strategy in the Bransford-Franks Paradigm. Manuscript submitted for publication. 1976.

\section{REFERENCES}

Bransford, J. D., \& Franks, J. J. The abstraction of linguistic ideas. Cognitive Psychology, 1971, 2, 331-350.

BRANSFORD, J. D., \& Franks, J. J. The abstraction of linguistic ideas: A review. Cognition, 1973, 1, 211-249.

FlagG, P. W., Potts, G. R., \& Reynolds, A. G. Instructions and response strategies in recognition memory for sentences. Journal of Experimental Psychology: Human Learning and Memory, 1975, 1, 592-598.

KATZ, S. Role of instructions in abstraction of linguistic ideas. Journal of Experimental Psychology, 1973, 98, 79-84.

Katz, S., Atkeson, B., \& LeE, J. The Bransford-Franks linear effect: Integration or artifact? Memory \& Cognition. 1974, 2, 709-713.

Katz, S., \& Gruenewald, P. The abstraction of linguistic ideas in "meaningless" sentences. Memory \& Cognition, 1974, 2. 737-741.

Reitman, J. S., \& Bower, G. H. Storage and later recognition of exemplars of concepts. Cognitive Psychology, 1973, 4, 194-206.

(Received for publication October 26, 1976.) 